\author{
Н.К. Калжигитов ${ }^{1}$ В.О. Курмангалиева ${ }^{1}$, Н.К. Аманжол ${ }^{1}$, Б.К. Тураров ${ }^{1}$ \\ ${ }^{1}$ Казахский национальный университет имени аль-Фараби, г. Алматы, Қазахстан

\section{ПРИМЕНЕНИЕ ДВУХКЛАСТЕРНОЙ МИКРОСКОПИЧЕСКОЙ МОДЕЛИ ДЛЯ ИССЛЕДОВАНИЯ ПРОЦЕССОВ, СВЯЗАННЫХ С КОСМОЛОГИЧЕСКОЙ ЛИТИЕВОЙ ПРОБЛЕМОЙ}

\begin{abstract}
Аннотация
В данной работе приведены результаты исследований долгоживущих резонансных состояний ядра ${ }^{6} \mathrm{Li}$ и реакции радиационного захвата, приводящих к синтезу ${ }^{6} \mathrm{Li}$. В связи с важностью литиевой проблемы для областей ядерной физики и ядерной астрофизики реакции первичного нуклеосинтеза представляют огромный интерес для изучения и рассмотрения с применением новых методов расчетов. Кластерные модели являются мощным инструментом для теоретического анализа и решения указанной проблемы. Одной из таких моделей является микроскопическая кластерная модель или метод, известный как алгебраическая версия метода резонирующих групп (АВМРГ), которая позволит исследовать данную задачу с новой стороны и получить данные о резонансных состояниях.

Для данной задачи был выбран модифицированный потенциал Хасегавы-Нагаты, который обладает своими уникальными характеристиками и обменными параметрами. Полученные данные сравнивались с экспериментальными данными и показали хорошее соответствие, и применимость данной методики к описанию подобных реакций, берущих свое начало в первые три минуты после рождения вселенной.

Ключевые слова: кластеры, кластерная модель, метод резонирующих групп, легкие ядра, реакция радиационного захвата, резонансные состояния.

\section{Анцдатnа \\ ЕКІ КЛАСТЕРЛІК МИКРОСКОПИЯЛЫҚ МОДЕЛДІ ҒАРЫШТЫК ЛИТИЙЛІК МӘСЕЛЕМЕН БАЙЛАНЫСКАН ПРОЦЕССТЕРДІ ЗЕРТТЕУГЕ КОЛДАНУ \\ Н.К. Калжигитов ${ }^{l}$, В.О. Курмангалиева ${ }^{1}$, Н.К. Аманжол ${ }^{1}$, Б.К. Тураров ${ }^{1}$ \\ ' әл-Фараби атындавы Қазақ ұлттық университеті, Алматы қ.., Қазақстан}

Бұл жұмыста ұзақ өмір сүретін ${ }^{6} \mathrm{Li}$ ядросының резонанстық күйлерін және ${ }^{6} \mathrm{Li}$ ядросының синтезіне әкелетін радиациялық қарпу реакцияларын зерттеулер нәтижелері келтірілген. Литий мәселесінің ядролық физика және ядролық астрофизика салалары үшін маңыздылығына байланысты, алғашқы нуклеосинтез реакциялары жаңа есептеу әдістерін қолдана отырып зерттеу және қарастыру үшін үлкен қызығушылық тудырады. Кластерлік моделдер аталған проблеманы шешуде және теориялық талдау жасау үшін күшті құрал болып табылады. Осындай моделдердің бірі - бұл мәселені жаңа қырынан зерттеуге және резонанстық күйлері туралы мәліметтер алуға мүмкіндік беретін, микроскопиялық кластерлік модель немесе резонацияланатын топтар әдісінің алгебралық нұсқасы (РТӘАН) әдісі.

Ядролық күштердің табиғатын зерттеу үшін, бұл есепке өзіндік ерекше сипаттамалары мен алмасу параметрлері бар, модификацияланған Хасегава-Нагата потенциалы таңдалды. Алынған мәліметтер эксперименттік мәліметтермен салыстырылды және жақсы сәйкес келді,осы әдістеме ғалам пайда болғаннан кейінгі алғашқы үш минутта басталған осындай ракцияларды сипаттауда қолдануға болатындығын көрсетті.

Түйін сөздер: кластерлер, кластерлік модель, резонациялайтын топтар әдісі, жеңіл ядролар, радиациялық қарпу реакциясы, резонанстық күйлер.

\title{
APPLICATION OF TWO-CLUSTER MICROSCOPIC MODEL TO STUDY PROCESSES ASSOCIATED WITH THE COSMOLOGICAL LITHIUM PROBLEM \\ Kalzhigitov N.K. ${ }^{1}$, Kurmangaliyeva V.O. ${ }^{1}$, Amanzhol N.K. ${ }^{1}$, Turarov B.K. ${ }^{1}$ ${ }^{I}$ Al-Farabi Kazakh National University, Almaty, Kazakhstan
}

This paper presents the results of studies of long-lived resonance states of the ${ }^{6} \mathrm{Li}$ nucleus and the radiative capture reaction leading to the synthesis of ${ }^{6} \mathrm{Li}$. In connection with the importance of the lithium problem for the fields of nuclear physics and nuclear astrophysics, the reactions of primary nucleosynthesis are of great interest for studies and consideration using new calculation methods. Cluster models are a powerful tool for theoretical analysis and solution of this problem. One of these models is the microscopic cluster model, or a method known as the algebraic version of the resonant group method (AVRGM), which will allow us to investigate this problem from a new angle and obtain data on resonance states. 
For this task, a modified Hasegawa-Nagata potential was chosen, which has its own unique characteristics and exchange parameters. The obtained data were compared with experimental data and it was shown that they showed good agreement, and the applicability of this technique to the description of similar reactions that originate in the first three minutes after the birth of the universe.

Keywords: clusters, cluster model, resonating group method, light nuclei, capture reaction, resonance states.

На сегодняшний день в области ядерной физики и ядерной астрофизики одним из интереснейших открытых вопросов является литиевая проблема, берущая свое начало из теории первичного нуклеосинтеза [1]. Данная теория еще именуется теорией нуклеосинтеза большого взрыва (Big bang nucleosynthesis $(\mathrm{BBN}))$ и рассматривает происхождение первичных легких элементов во вселенной. К ним относятся: изотоп водорода - t, $\mathrm{d}$, гелия $-{ }^{3} \mathrm{He},{ }^{4} \mathrm{He}$, а также лития $-{ }^{6} \mathrm{Li},{ }^{7} \mathrm{Li}$ в начальный период появления вселенной от 1 секунды до 3 минут после большого взрыва. С использованием экспериментальных данных полученных с помощью системы WMAP (Wilkinson Microwave Anisotropy Probe) теория первичного нуклеосинтеза выстроила надежные теоретические прогнозы по возможному содержанию описываемых элементов во вселенной. Предсказанные теорией данные о количественном содержании легких элементов и в особенности для изотопов водорода: $\mathrm{d}$ дейтерия и гелия 4 Не показали хорошее соответствие с информацией от WMAP, став одной из самых успешных теории в описании зарождения вещества во вселенной. Однако вместе с этим данная теория столкнулась с несколькими проблемами связанными в появившихся расхождениях между данными с WMAP и теоретическими прогнозами для изотопов лития ${ }^{6} \mathrm{Li},{ }^{7} \mathrm{Li}$. Данные с WMAP для изотопов ${ }^{6} \mathrm{Li}$, ${ }^{7} \mathrm{Li}$ неожиданно получились в 3-4 раза ниже, чем предполагала теория первичного нуклеосинтеза. В следствии этого появился вопрос о предположительной концентрации изотопов лития в старых звездах, которые по нынешним экспериментальным данным значительно уступали концентрации предсказываемой теорией $\mathrm{BBN}$ и той, что была в более новых звездах. Данная проблема получила название - Изначальная литиевая проблема [2].

Как ${ }^{7} \mathrm{Li}$, изотоп ${ }^{6} \mathrm{Li}$ является одним из немногих элементов, появившихся в процессе первичного нуклеосинтеза в ранние этапы вселенной. Так изотоп ${ }^{7} \mathrm{Li}$ появился в количестве не более чем $10^{-9}$ от всех элементов. Изотоп ${ }^{6} \mathrm{Li}$ появился в заметно меньшем количестве по сравнению ${ }^{7} \mathrm{Li}$, уступая ему как минимум в десять тысяч раз, что показывает большую распространенность ${ }^{7} \mathrm{Li}$ как первичного изотопа.

$$
\frac{{ }_{3}^{6} \mathrm{Li}}{7 \mathrm{Li}}=0.05
$$

Однако, полученные наблюдения также показывают существование ${ }^{6} \mathrm{Li}$, хоть и в довольно меньших количествах, который также мог образоваться в процессе первичного нуклеосинтеза. Данные вопросы являются крайне важным и входят в ряд основных проблем исследования ядерной астрофизики.

Для исследования литиевой проблемы была выбрана реакция радиационного захвата ${ }^{6} \mathrm{Li}$, что по теории первичного нуклеосинтеза имеет место быть в момент первичного нуклесинтеза.

$$
\alpha+\mathrm{d} \rightarrow{ }_{3}^{6} \mathrm{Li}+\gamma .
$$

Представленная реакция рассматривалась с позиции кластерного представления и с учетом особенностей кластерной модели. Для расчетов был использован нуклон-нуклонный потенциал Хасегавы-Нагаты и микроскопический метод - Алгебраической версии метода резонирующих групп (АВМРГ). Используемая методика в полной мере позволяла учесть принцип Паули и внутреннюю структуру исследуемых ядер, рассматриваемых с позиции кластеров. В рамках этого метода ядро ${ }^{6} \mathrm{Li}$ было рассмотренно как состоящее из двух кластеров: альфа частицы и дейтрона. Волновые функции ядра ${ }^{6} \mathrm{Li}$ для состояний дискретного и непрывного спектров находились в результате решения многочастичного уравнения Шредингера.

$$
\widehat{H} \Psi=E \Psi,
$$

где $\widehat{H}$ - оператор гамильтониана, $\mathrm{E}$ - полная энергия системы, а $\Psi$ - волновая функция. С учетом кластерной структуры исследуемого ядра многочастичное уравнение Шредингера сводится к двухчастичному уравнению с нелокальным потенциалом взаимодействия кластеров. Решение 
двухчастичного уравнения Шредингера позволяет определить все величины, описывающие интересующие нас состояние ядра и происходящие в нем процессы. Однако для решения уравнения Шредингера нужно определить достаточно точно вид нуклон-нуклонного потенциала и получить приближенное решение уравнения Шредингера для А тел. В следствии чего в обычном случае данная задача значительно усложняется из-за того, что объектом исследования являются фермионы и при этом должен выполняться принцип Паули. Учет принципа Паули в расчетах непременно приводит к появлению в волновой функции системы оператора антисимметризации $\hat{A}$, поэтому для двухкластерой системы она принимает вид:

$$
\Psi_{J}=\hat{A}\left\{\left[\varphi_{1}\left(A_{1}\right) \varphi_{2}\left(A_{2}\right)\right]_{s} \psi_{L S}^{J}(\vec{q})\right\} .
$$

В представленном выражении для полной волновой функции двухкластерной системы $\Psi_{J}$ компоненты $\varphi_{1}\left(A_{1}\right)$ и $\varphi_{2}\left(A_{2}\right)$ представляются внутренней волновой функцией первого и второго исследуемого кластера данной ядерной системы, а $A_{1}$ и $A_{2}$ непосредственно их массовые числа. В свою очередь $\psi_{L S}^{J}(\vec{q})$ является волновой функцией относительного движения этих двух кластеров и определяется с помощью вектора Якоби.

$$
\vec{q}=\sqrt{\frac{A_{1} A_{2}}{A_{1}+A_{2}}}\left[\frac{1}{A_{1}} \sum_{i \in A_{1}} \vec{r}_{i}-\frac{1}{A_{2}} \sum_{J \in A_{2}} \vec{r}_{j}\right]
$$

где $\vec{r}_{i}$ и $\vec{r}_{j}$ - это координаты нуклонов из первого и второго кластеров.

Для определения полной волновой функции данной системы $\Psi_{J}$ и решения уравнения Шредингера с учетом оператора антисимметризации $\hat{A}$ использован микроскопический метод алгебраической версии метода резонирующих групп (АВМРГ) [3-5]. Отличием данного метода от классической версии метода резонирующих групп [6] состоит в том, что представленный метод позволял обходить сложные вычисления интегрально-дифференциального уравнения Хилла-Уилера [7, 8], которые проявлялись из-за влияния оператора антисимметризации. Метод АВМРГ упрощает аналитические и численные вычисления и сводит их к простой алгебраической форме благодаря разложению межкластерной функции по полной системе осцилляторных функций.

Данная форма (версия) метода резонирующих групп, которую из-за своих особенностей формы уравнений принято называть алгебраической версией МРГ была предложена профессором Г.Ф. Филипповым [3-5] и эффективно использовалась для исследования структуры атомных ядер и динамики разнообразных процессов.

В алгебраической версии метода резонирующих групп полная волновая функция двухкластерной системы (4) принимает вид обобщенного ряда Фурье

$$
\Psi_{J}=\sum_{n=n_{0}}^{\infty} \mathrm{C}_{n L} \Psi_{n L},
$$

где $\mathrm{C}_{n L}$ - коэффициент разложения по базисным функциям $\Psi_{\mathrm{nL}}$. Метод АВМРГ и детали расчетов были описаны в работах [9-11]. Учитывая разложение (6), уравнение Шредингера преобразуем к следующему виду:

$$
\sum_{m=n_{0}}^{\infty}\left[\langle\bar{n} L|\widehat{\mathrm{H}}| \bar{m} L\rangle-\mathrm{E} \cdot \delta_{n, m}\right] \overline{\mathrm{C}}_{m L}=0,
$$

где $\langle\bar{n} \mathrm{~L}|\widehat{\mathrm{H}}| \bar{m} L\rangle$ - матричные элементы оператора гамильтона на функциях $\Psi_{n L}, \mathrm{n}$ и $\mathrm{m}$ - номера кластерных осцилляторных функций, являющиеся индексами и нумеруют только состояния разрешенные принципом Паули.

Данная форма уравнения является стандартной матричной формой уравнения Шредингера с ортонормированным базисом функции, что порождает бесконечную систему линейных однородных алгебраических уравнений. Если учесть асимптотическое поведение коэффициентов разложения, то система уравнений (7) примет вид

$$
\begin{gathered}
\sum_{m=n_{0}}^{\mathrm{N}_{a}}\left[\langle\bar{n} L|\widehat{\mathrm{H}}| \bar{m} L\rangle-\mathrm{E} \cdot \delta_{n, m}\right] \overline{\mathrm{C}}_{m L}-\tan \delta \cdot\left\langle\bar{n} L|\widehat{\mathrm{H}}| \mathrm{N}_{a}+1, L\right\rangle \mathrm{C}_{\mathrm{N}_{a}+1, L}^{(I)}= \\
=-\left\langle\bar{n} L|\widehat{\mathrm{H}}| \mathrm{N}_{a}+1, L\right\rangle \mathrm{C}_{\mathrm{N}_{a}+1, L}^{(R)} .
\end{gathered}
$$


Это конечная система линейных уравнений, она состоит из $\mathrm{N}_{a}+1$ уравнений и содержит $\mathrm{N}_{a}$ коэффициетов разложения внутренней части волновой функции и тангенс фазы рассеяния. При решении данная неоднородная система линейных алгебраических уравнений с граничными условиями позволяет найти волновую функцию и фазу рассеяния непрерывного спектра в осцилляторном представлении.

Для описания ядерного взаимодействия представленной кластерной системы использовался модифицированный потенциал Хасегавы-Нагаты (MHNP) [12, 13], учитывающий, как центральную, так и спин-орбитальную компоненту. Данный потенциал содержит в себе 3 гауссовских функции:

$$
V(i j)=V_{0} \exp \left\{-\left(\frac{r_{i}-r_{j}}{a_{v}}\right)^{2}\right\}
$$

где $V_{0}-$ глубина потенциальной ямы или интенсивность взаимодействия, $a$ - радиус соответствующего потенциала. Исспользуемый потенциал воспроизводит притяжение нуклонов на больших расстояниях и отталкивание на малых расстояниях. Он часто привлекается для описания данных по рассеянию легких ядер в широком диапазоне энергий. Содержащиеся в потенциале компоненты для центральной и спин-орбитальной части потенциала выражаются через обменные операторы и обменные параметры.

Центральная компонента модифицированного потенциала Хасегавы-Нагаты имеет вид

$$
V_{c}\left(r_{i j}\right)=\sum_{n=1}^{3}\left(w_{c}^{(n)}+m_{c}^{(n)} P_{i j}^{M}+b_{c}^{(n)} P_{i j}^{B}+h_{c}^{(n)} P_{i j}^{H}\right) V_{C o}^{(n)} e^{\left(-\mu_{c}^{(n)} r_{i j}^{2}\right)}
$$

Спин-орбитальная компонента модифицированного потенциала Хасегавы-Нагаты равна

$$
V_{L S}\left(r_{i j} ; \sigma_{i}, \sigma_{j}\right)=\sum_{n=1}^{2}\left(w_{L S}^{(n)}+m_{L S}^{(n)} P_{i j}^{M}\right) V_{L S o}^{(n)} e^{\left(-\mu_{L S}^{(n)} r_{i j}^{2}\right)}
$$

где $\mathrm{n}$ - номер гауссовской функции, $\mu$ - параметр, обратный радиусу сил в квадрате, $\sigma_{i}$ - оператор спина і-го нуклона, $\tau_{i}$ - изоспиновая переменная, обменные операторы ( $P^{M}$-Майорана, $P^{B}-$ Бартлетта, $P^{H}$-Гейзенберга), обменные параметры ( $h$-Гейзенберга, $b$-Бартлетта, $\mathrm{m}$ - Майорана), $w-$ параметр Вигнера. Параметры: $w, b, h, \mathrm{~m}$ - являются безразмерными и изменяются в пределах от 0 до 1. Их значения были определены подгонкой энергии связи дейтрона и параметров нуклоннуклонного рассеяния. В таблице 1 приведены явные значения параметров $w, b, h, \mathrm{~m}$ для центральных компонент модифицированного потенциала Хасегавы-Нагаты

Таблица 1. Значения обменных параметров для модифищированного потенииала Хасегавы-Нагаты [12, 13]

\begin{tabular}{|c|c|c|c|c|c|c|c|}
\hline Потенциал & $n$ & $\mu\left(\mathrm{pm}^{-2}\right)$ & $V_{0}(\mathrm{MэB})$ & $m$ & $w$ & $b$ & $h$ \\
\hline \multirow{2}{*}{ МНNP } & 1 & 0.16 & -6 & 1.1528 & -0.2361 & 0.5972 & -0.5139 \\
& 2 & 1.127 & -546 & 0.4057 & 0.424 & 0.1401 & 0.0302 \\
& 3 & 3.4 & 1655 & 0.3985 & 0.4474 & 0.1015 & 0.0526 \\
\hline
\end{tabular}

После того как был выбран нуклон-нуклонный потенциал, необходимо определить входные параметры модели. Осцилляторный радиус $b$ был выбран из условия минимума энергии порога канала $\alpha+\mathrm{d}$. Кроме этого, была выпонена небольшая подгонка параметра Майорана $m$ и интенсивности спин-орбитального взаимодействия $f_{L S}$. Эта подгонка позволила получить близкую к эксперимету энергию связи ${ }^{6} \mathrm{Li}$. В таблице 2 приведены все входные параметры модели и их конкретные значения, где $b$ - осцилляторная длина, $\Delta m$ - величина отклонения от исходного параметра Майорана, $f_{L S}$ - интенсивность спин-орбитального взаимодействия, $E$ - энергия связанного состояния ${ }^{6} \mathrm{Li}$ относительно порога $\alpha+\mathrm{d}$.

Таблица 2. Основные входные параметры расчетов и энергия основного состояния ${ }^{6} \mathrm{Li}$.

\begin{tabular}{|l|c|c|c|c|}
\hline Потенцииал & $b(\mathrm{fm})$ & $\Delta m$ & $f_{L S}$ & $E($ МэB $)$ \\
\hline MHNP & 1.357 & -0.0009 & 0.348 & -1.473 \\
\hline
\end{tabular}


Полученные для радиационного захвата (2) сечения реакции и фазы упругого $\alpha+\mathrm{d}$ рассеяния строились для значений полного углового момента $\mathrm{J}^{\pi}=1^{+}, 2^{+}, 3^{+}$в отрезке энергий от 0 до 10 МэВ.

Анализируя рисунок (1) мы видим, что долгоживущий $3^{+}$резонанс дает огромный вклад в сечение радиационного захвата. Этот резонанс проявляет себя в очень узкой области энергий, соразмерной его ширине. Вклад более широких резонансов $1^{+}$и $2^{+}$в парциальные и полное сечения не такой большой как вклад $3^{+}$резонанса.

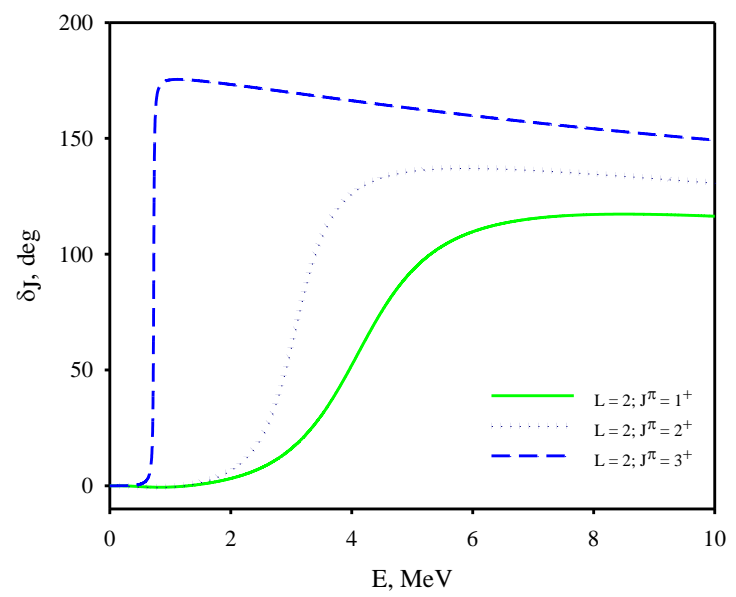

Рисунок 1. Фазы упругого $\alpha+d$ рассеяния в состояниях с орбитальным моментом $L=2$, но с разными значениями полного углового момента Ј. Результаты получены с использованием модифицированным потенцииалом Хасегавы-Нагаты.

Полученные графики фаз рассеяния (рисунок 1) и сечений рассеяния (рисунок 2) четко показали наличие резонансов $1^{+}, 2^{+}$, и $3^{+}$у ядра ${ }^{6} \mathrm{Li}$. Поскольку расчеты сечений радиационного захвата проходили в длинноволновом приближении, то дипольный переход из непрерывного спектра в основное состояние ядра ${ }^{6} \mathrm{Li} \mathrm{не} \mathrm{принимал} \mathrm{участия.} \mathrm{Это} \mathrm{связанно} \mathrm{с} \mathrm{тем,} \mathrm{что} \mathrm{дипольные} \mathrm{переходы} \mathrm{в}$ длинноволновом приближении запрещены для ядер с одинаковым числом нейтронов и протонов, к которым и относилось исследуемое ядро ${ }^{6} \mathrm{Li}$. В связи с этим все расчеты сечения радиационного захвата были выполнены только для квадрупольного перехода (таблица 3).

Таблииа 3. Экспериментальные и теоретические значения параметров резонансных состояний ${ }^{6} \mathrm{Li}$

\begin{tabular}{|l|c|c|c|}
\hline Потенциал & $L ; J^{\pi}$ & $E(M э B)$ & $\Gamma(M \ni B)$ \\
\hline МНNP & $2 ; 1^{+}$ & 4.100 & 2.357 \\
& $2 ; 2^{+}$ & 3.063 & 1.013 \\
& $2 ; 3^{+}$ & 0.763 & 0.019 \\
\hline Экспериментальные данные & $2 ; 1^{+}$ & $4.176 \pm 0.050$ & $1.5 \pm 0.2$ \\
{$[14,15]$} & $2 ; 2^{+}$ & $2.838 \pm 0.022$ & $1.30 \pm 1.00$ \\
& $2 ; 3^{+}$ & $0.712 \pm 0.002$ & $0.024 \pm 0.002$ \\
\hline
\end{tabular}

Как показали теоретические и экспериментальные данные (таблица 3) в области низких энергий от 500 до 900 кэВ только для состояния $3^{+}$проявляется и преобладает узкий резонанс, который при значениях $\mathrm{E}_{\mathrm{MHNP}}=763$ кэВ для модифицированного потенциала Хасегавы-Нагаты, хорошо согласовывается с экспериментальными данными при которых $\mathrm{E}_{\text {exp. }}=711$ кэВ. Данные значения энергии соответствуют первому возбужденному состоянию ядра ${ }^{6} \mathrm{Li}$ со значением энергии $\mathrm{E}_{\text {возб }}=$ 2.186 МэВ.

Ширина данного узкого резонанса равна $\Gamma_{\text {мнNP }}=19$ кэВ для модифицированного потенциала Хасегавы-Нагаты, что также соответствует экспериментальным данным, для которых ширина резонанса равна $\Gamma_{\text {exp. }}=24-26$ кэВ. В следствии полученных данных можно утверждать, что в состояниях с полным угловым моментом $\mathrm{L}=2$ существует достаточно мощный барьер, образованный центробежными силами и кулоновским потенциалом. 


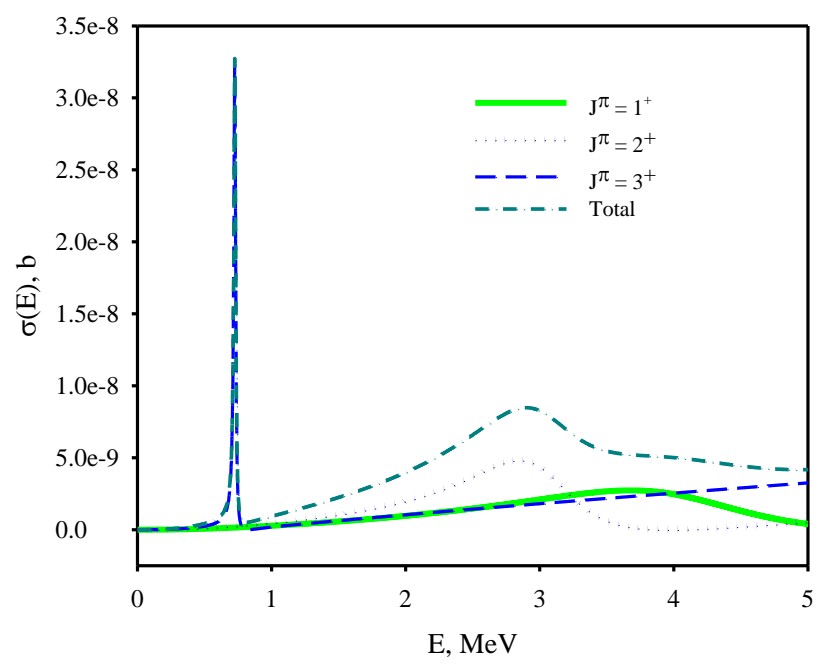

Рисунок 2. Полное и парцииальные сечения радиациионого захвата $\alpha+d \rightarrow{ }_{3}^{6} \mathrm{Li}+\gamma$, порождаемые квадрупольными переходами из состояний непрерывного $\alpha+d$ спектра в основное $1^{+}$состояние ядра ${ }^{6} \mathrm{Li}$. Результаты получень с использованием модифицированньм потеничиалом Хасегавы-Нагать

Это в свою очередь приводит к туннелированию дейтронов в реакции радиационного захвата $\alpha+\mathrm{d}$ $\rightarrow{ }^{6} \mathrm{Li}+\gamma$. Этот барьер порождает, например, долгоживущий $3^{+}$резонанс с временем жизни порядка $2-3 \cdot 10^{-20}$ с, что приводит к существенному увеличению сечения реакции радиационного захвата.

Для состояний $1^{+}$и $2^{+}$полученные теоретические результаты, как и экспериментальные данные, также показали наличие резонансов, однако в отличии от случая с $3^{+}$состоянием, рассчитанные резонансы оказались более широкими и кратко живущими, чем экспериментальные.

\section{Заключение}

Структура ядра ${ }^{6} \mathrm{Li}$ исследована в рамках алгебраической версии метода резонирующих групп. Нуклон-нуклонное взаимодействие моделировалось полуреалистичным потенциалом ХасегавыНагаты. Детально иследованы связанное состояние ядра ${ }^{6} \mathrm{Li}$, а также непрерывный спектр свяанный с рассеянием дейтронов на альфа-частице. В рамках используемой модели, все обнаруженные резонансы ядра ${ }^{6} \mathrm{Li}$ имеют значение орбитального момента $\mathrm{L}=2$, что свидетельствует о наличии в данных состояниях достаточно мощного центробежного барьера, который также дополняет кулоновский барьер. Спин-орбитальные силы увеличивают притяжение в состоянии с полным угловым моментом и четностью $\mathrm{J}^{\pi}=3^{+}$и порождают долгоживущий резонанс с наименьшей шириной Г при малых энергиях. В состоянии $\mathrm{J}^{\pi}=1^{+}$эти силы напротив уменьшают притяжения альфа-частицы с дейтроном и выталкивают резонанс на сравнительно большую энергию, где очень слабый барьер и его ширина максимальна. Спин-орбитальное взаимодествие слабо влияет на состояние $\mathrm{J}^{\pi}=2^{+}$и его ширина резонанса меньше, чем у $1^{+}$резонанса, но больше чем $3^{+}$резонанса.

Кроме этого показано, что результаты расчетов в рамках используемой двухкластерной модели находятся в хорошем согласии с имеющими экспериментальными данными, а также согласуются с результатами других микроскопических моделей.

\section{Благодарность}

Авторы статьи выражают благодарность В.С.Василевскому за обсуждение полученных результатов и советов при написании статьи.

Список использованной литературы:

1 Iocco F., Mangano G., Miele G. Primordial nucleosynthesis: from precision cosmology to fundamental physics, Phys. Rep. - 2009. - Vol. 472. - P. 1-76.

2 Brian D. Fields. The Primordial Lithium Problem, Annual Review of Nuclear and Particle Science. - 2011. Vol. 61. - P. 47-68.

3 Филиппов Г.Ф., Охрименко И.П. О возможности использования осцилляторного базиса для решения задач непрерывного спектра, ЭЧАЯ. - 1980. - Том. 32. - С. 5. 
4 Filippov G. F. "On taking into account correct asymptotic behavior in oscillator-basis expansions," Sov. J. Nucl. Phys., vol. 33, pp. 488-489, 1981.

5 Филиппов Г.Ф., Василевский В.С., Чоповский Л.Л. Обобщенные когерентные состояния в задачах ядерной физики, ЭЧАЯ. - 1984. - Т.15. - стр.1338-1383.

6 Wheeler. J.A. On the mathematical description of light nuclei by the method of resonating group structure, Phys. Rev. - 1937. - vol. 52. - pp. 1107-1122.

7 Hill D.L., Wheeler J.A., "Nuclear continuation and the interpretation of fission phenomena”, Phys. Rev., vol. 89, pp. 1102_1145, 1953.

8 Griffin J.J. and Wheeler J.A., "Collective motions in nuclei by the method of generator coordinates", Phys. Rev., vol. 108, pp. 311-327, 1957.

9 Filippov G. F., Vasilevsky V. S., and Chopovsky L. L., "Solution of problems in the microscopic theory of the nucleus using the technique of generalized coherent states”, Sov. J. Part. Nucl., vol. 16, pp. 153-177, 1985.

10 Vasilevsky V. S., Kato K., Kurmangaliyeva V., Duisenbay A.D., Kalzhigitov N., Takibayev N., Investigation of discrete and continuous spectrum states in two-cluster system. Sapporo, Japan: Hokkaido University, 2017.

11 Lashko Y. A., Filippov G. F., and Vasilevsky V. S., "Dynamics of two cluster systems in phase space”, Nucl. Phys. A, vol. 941, pp. 121-144, Sept. 2015.

12 Hasegawa A. and Nagata S., "Ground state of ${ }^{6}$ Li", Prog. Theor. Phys., vol. 45, pp. 1786-1807, 1971.

13 Tanabe F., Tohsaki A., Tamagaki R., “ $\alpha \alpha$ scattering at intermediate energies”, Prog. Theor. Phys., vol. 53, pp. 677-691, 1975.

14 Tilley D. R., Cheves C. M., Godwin J. L., Hale G. M., Hofmann H. M., Kelley J. H., Sheu C. G., Weller H. R. “Energy levels of light nuclei A=5, 6, 7”, Nuclear Physics A, vol. 708, pp. 3-163, 2002.

15 Robertson R. G. H., Dyer P., Warner R. A., "Observation of the Capture Reaction ${ }^{2} H(\alpha, \gamma)^{6} L i$ and Its Role in Production of ${ }^{6} \mathrm{Li}$ in the Big Bang”, Phys. Rev. Lett, vol. 47, pp. 1867-1870, 1981.

\section{References:}

$1 \quad$ Iocco F., Mangano G., Miele G. (2009) Primordial nucleosynthesis: from precision cosmology to fundamental physics, Phys. Rep. Vol. 472, 1-76. [In English]

2 Brian D. Fields. (2011) The Primordial Lithium Problem, Annual Review of Nuclear and Particle Science. Vol. 61, 47-68. [In English]

3 Filippov G.F., Ohrimenko I.P. (1980) O vozmozhnosti ispol'zovanija oscilljatornogo bazisa dlja reshenija zadach nepreryvnogo spektra, JeChAJa [On the possibility of using the oscillatory basis to solve the tasks of the negative spectrum]. Том.32, 5. (In Russian)

4 Filippov G. F. (1981) "On taking into account correct asymptotic behavior in oscillator-basis expansions," Sov. J. Nucl. Phys., vol. 33, 488-489. [In English]

5 Filippov G.F., Vasilevskij B.C., Chopovskij L.L.(1984) Obobshhennye kogerentnye sostojanija v zadachah jadernoj fiziki, JeChAJa.T.15,1338-1383. (In Russian)

6 Wheeler. J.A. (1937) On the mathematical description of light nuclei by the method of resonating group structure, Phys. Rev.vol. 52, 1107-1122. [In English]

7 Hill D.L., Wheeler J.A. "Nuclear continuation and the interpretation of fission phenomena", Phys. Rev., vol. 89, 1102, 1145, 1953. [In English]

8 Griffin J.J. and Wheeler J.A.(1957) "Collective motions in nuclei by the method of generator coordinates", Phys. Rev., vol. 108, 311-327. [In English]

9 Filippov G. F., Vasilevsky V. S., and Chopovsky L. L. (1985) "Solution of problems in the microscopic theory of the nucleus using the technique of generalized coherent states”, Sov. J. Part. Nucl., vol. 16,153-177. [In English]

10 Vasilevsky V. S., Kato K., Kurmangaliyeva V., Duisenbay A.D., Kalzhigitov N., Takibayev N. (2017) Investigation of discrete and continuous spectrum states in two-cluster system. Sapporo, Japan: Hokkaido University.

11 Lashko Y. A., Filippov G. F., and Vasilevsky V. S. (2015) "Dynamics of two cluster systems in phase space”, Nucl. Phys. A, vol. 941, 121-144. [In English]

12 Hasegawa A. and Nagata S. (1971) "Ground state of Li”, Prog. Theor. Phys., vol. 45, 1786-1807. [In English]

13 Tanabe F., Tohsaki A., Tamagaki R. (1975) “Aa scattering at intermediate energies”, Prog. Theor. Phys., vol. 53,677-691. [In English]

14 Tilley D. R., Cheves C. M., Godwin J. L., Hale G. M., Hofmann H. M., Kelley J. H., Sheu C. G., Weller H.R. (2002) “Energy levels of light nuclei A=5, 6, 7”, Nuclear Physics A, vol. 708, 3-163 [In English].

15 Robertson R. G. H., Dyer P., Warner R. A. (1981) "Observation of the Capture Reaction ${ }^{2} H(\alpha, \gamma)^{6} \mathrm{Li}$ and Its Role in Production of ${ }^{6}$ Li in the Big Bang”, Phys. Rev. Lett, vol. 47,1867-1870. [In English] 